\title{
La collaboration de Pareto avec la Gazette de
}

Lausanne

Fiorenzo Mornati

\section{OpenEdition}

\section{Journals}

Édition électronique

URL : http://journals.openedition.org/ress/756

DOI : $10.4000 /$ ress. 756

ISSN : 1663-4446

Éditeur

Librairie Droz

Édition imprimée

Date de publication : 1 juillet 2010

Pagination : 71-88

ISBN : 978-2-600-01449-6

ISSN : 0048-8046

Référence électronique

Fiorenzo Mornati, «La collaboration de Pareto avec la Gazette de Lausanne », Revue européenne des sciences sociales [En ligne], XLVIII-146 | 2010, mis en ligne le 01 juillet 2013, consulté le 03 mai 2019.

URL : http://journals.openedition.org/ress/756 ; DOI : 10.4000/ress.756 


\section{Fiorenzo MORNATI}

\section{LA COLLABORATION DE PARETO AVEC LA GAZETTE DE LAUSANNE}

Dans cette étude, après avoir rappelé la ligne politico-éditoriale de la Gazette de Lausanne de l'époque $(\$ 1)$ et les raisons intellectuelles de la collaboration de Pareto au quotidien lausannois $(\$ 2)$, nous reconstruirons $(\$ 3)$ les contenus de cette collaboration, qui dure une dizaine d'années et s'étend sur une quarantaine d'articles, en la replaçant dans le cadre de la biographie intellectuelle de l'économiste sociologue.

\section{LA GAZETTE DE LAUSANNE À L'ÉPOQUE DE LA COLLABORATION DE PARETO}

Dans son ouvrage fondamental sur l'histoire de la Gazette de Lausanne, Alain Clavien situe la collaboration de Pareto avec le quotidien lausannois dans le cadre de l'ouverture aux universitaires lausannois entamée par la Gazette dès la moitié des années 1890. Cette démarche constituait l'un des aspects de la réforme lancée par son rédacteur en chef, Edouard Secrétan, et était destinée à faire de ce journal la référence du public cultivé de la Suisse entière ${ }^{1}$. L'objectif fut atteint au début du siècle au moins en Suisse romande ${ }^{2}$.

En fait, jusqu'à ce moment-là, la Gazette s'était limitée à s'engager, en tant qu'organe officieux du parti libéral vaudois, dans la lutte politique lausannoise et cantonale. Avec des résultats modestes, quand bien même la paix avait été signée, en 1892, avec le parti radical, alors dominant ${ }^{3}$ : un événement qui, selon l'interprétation de Clavien, était à l'origine de la politique éditoriale novatrice susmentionnée ${ }^{4}$.

Il est intéressant de s'arrêter un moment sur le type de libéralisme soutenu par la Gazette dans les questions économiques et sociales, qui seront celles traitées par Pareto. Jusqu'à la moitié des années 1880, la Gazette avait prôné l'idée de l'identité entre laissez-faire et science économique. Avec, comme corollaire, la

1 Alain Clavien, Histoire de la Gazette de Lausanne. Le temps du Colonel, Vevey, Editions de l'Aire, 1997, p. 199.

2 Ibidem, pp. 124, 157, 163. Les autres professeurs dont Secrétan s'attache les services sont Edmond Rossier (historien), Henri Dufour (physicien), François-Alphonse Forel (limnologue), Maurice Millioud (théologien et futur sociologue), Louis Emery (théologien) et Léon Walras, ibidem, p. 157. Pareto, qui est payé 30 francs l'article, compte parmi les collaborateurs les mieux rémunérés de la Gazette, ibidem, p. 180.

3 Ibidem, pp. 116-118.

4 Ibidem, p. 124. 
conviction qu'il n'y avait pas de question sociale, mais que la misère n'était qu'un problème d'imprévoyance individuelle qu'il aurait fallu, tout au plus, résoudre par le biais de la charité chrétienne, c'est-à-dire privée et spontanée ${ }^{5}$.

A partir de ce moment-là, à la suite d'une vague de grèves en Europe, la Gazette quitte cette attitude dogmatique et décide de regarder de plus près ce système économique désormais caractérisé, à l'évidence, par le clivage entre le prolétariat et le grand capital $^{6}$. La Gazette confie alors la description du phénomène économique au publiciste bernois Constant Bodenheimer ${ }^{7}$ et l'étude de la politique sociale à l'ancien, mais prestigieux, philosophe et professeur de l'Université de Lausanne, Charles Secrétan. Celui-ci, à partir de son protestantisme libéral imprégné de fortes teintes sociales, développe une longue réflexion qui l'amène à reconnaître sans autre l'existence de la question sociale et à proposer qu'on lui apporte des remèdes, sous l'égide de la charité chrétienne mais aussi au nom de l'intérêt des capitalistes à éviter des émeutes sociales. Charles Secrétan souhaite notamment des mesures comme «[la] protection du travail des mineurs et des femmes, [le] repos hebdomadaire, [la] journée normale de huit heures, [les] assurances accidents $»^{8}$. Toutefois, selon Secrétan, la vraie réponse à la question sociale réside dans le remplacement du salariat par la coopération?

Cette période du libéralisme non dogmatique et socialisant de la Gazette, mais en tout cas toujours inflexible dans son attitude hostile au socialisme jugé autoritaire et matérialiste ${ }^{10}$, se termine assez vite avec le décès de Bodenheimer, en 1893, et de Secrétan, en $1895^{11}$.

Edouard Secrétan confie alors les chroniques économiques de la Gazette au nouveau correspondant bernois du journal, le publiciste fribourgeois Jules Répond, qui s'inscrit dans l'attitude antérieure de la Gazette et se déclare favorable, sans préoccupation sociale, au libéralisme économique pur et dur ${ }^{12}$. Ainsi, dix ans plus tard, la Gazette aura complètement réinvesti sa position traditionnellement libéraleconservatrice, selon laquelle «la misère est un problème strictement individuel ${ }^{13}$ : pour reprendre une polémique de l'époque, c'est l'alcoolisme qui serait la cause de la misère des prolétaires, et non le contraire, comme le prétendent les socialistes ${ }^{14}$.

\section{LES RAISONS DE LA COLLABORATION DE PARETO AVEC LA GAZETTE DE LAUSANNE}

Ceci rappelé, notre recherche vise à étudier non seulement les contenus, mais aussi les raisons de la collaboration de Pareto avec la Gazette, qui durera de 1893

\footnotetext{
5 Ibidem, pp. 88, 91.

${ }^{6}$ Ibidem, p. 90.

7 Ibidem, pp. 89-92.

${ }^{8}$ Ibidem, p. 96.

9 Ibidem, p. 96.

${ }^{10}$ Ibidem, p. 95.

${ }^{11}$ Ibidem, p. 126.

12 Ibidem, pp. 157-158.

13 Ibidem, p. 197.

${ }^{14}$ Ibidem, p. 198.
} 
à 1902. A cette date, Pareto, avec d'autres collaborateurs, passera au Journal de Genève, alléché par le meilleur traitement que lui propose Horace Micheli, le nouveau rédacteur en chef du quotidien genevois, qu'il cherche à relancer grâce à des signatures célèbres ${ }^{15}$.

Pour bien comprendre les raisons de la collaboration de Pareto avec la Gazette, il nous faut revenir sur les raisons mêmes qui ont poussé Pareto à Lausanne. Il faut en effet se souvenir que, depuis le printemps 1890, c'est-à-dire depuis sa houleuse démission de la direction de la forge de San Giovanni Valdarno (près de Florence) qu'il avait conservée pendant plus de quinze ans, Pareto s'était consacré à la défense du libre-échange par le biais de travaux en économie mathématique (qui lui avaient assuré le soutien de Walras) et d'une collaboration régulière avec les organes français et italiens de la petite, mais passionnée, patrouille libre-échangiste.

Pareto, à l'époque, est aussi de l'avis qu'il faut révéler les vérités de la science $^{16}$ et c'est la raison pour laquelle, en novembre $1892^{17}$, il accepte l'idée de Walras de le proposer pour repourvoir la chaire d'économie politique à la Faculté de droit de l'Université de Lausanne: il souhaite pouvoir exprimer en public ses idéaux et ses théories libre-échangistes mais ne possède pas les ressources matérielles indispensables pour le faire ${ }^{18}$. Son objectif, lié à la promotion du libreéchangisme, est confirmé, à mots couverts, par Pareto à son ami Teodoro Moneta (le futur récipiendaire du Prix Nobel de la paix en 1908) quelques jours avant son départ pour Lausanne ${ }^{19}$.

Aussi, dès son arrivée à Lausanne, la collaboration entre Pareto et la Gazette de Lausanne s'instaure-t-elle sous le signe d'une parfaite continuité dans la biographie intellectuelle de Pareto. Nous pourrions aussi dire que la collaboration avec la Gazette (mais aussi avec la Bibliothèque universelle et le Journal de Genève) constitue un complément de l'enseignement universitaire, placé au début sous l'égide du libéralisme, de Pareto.

\section{LA PENSÉE PARÉTIENNE TELLE QUE PROPOSÉE AUX LECTEURS DE LA GAZETTE}

Pour les lecteurs du quotidien lausannois qui, le 5 décembre 1893, lisent son premier article ${ }^{20}$, Pareto n'est pas un inconnu. Depuis le mois de mai professeur

15 Ibidem, pp. 164-165.

16 Vilfredo Pareto, Les théories économiques III, «Le Monde économique», 25.8.1894, maintenant dans idem, Marxisme et économie pure, Genève, Droz, 1987, pp. 75-78.

${ }^{17}$ Lettre de Pareto à Walras du 7 novembre 1892, dans V. Pareto, Epistolario, t. I, Genève, Droz, 1975, pp. 191-192.

${ }^{18}$ Lettre de Pareto à Walras du 13 mars 1893, ibidem, pp. 191-192. Il faut toujours garder à l'esprit aussi que Pareto fut en mesure d'accepter la proposition walrassienne parce qu'il était de langue maternelle française. Ce qui a été aussi, à notre avis, la raison fondamentale qui a poussé Eugène Ruffy et Georges Favey (recteur de l'Université) à donner à Pareto, alors publiciste largement inconnu, sa chance à Lausanne.

${ }^{19}$ Lettre de Pareto à Teodoro Moneta du 2 mai 1893, dans V. Pareto, Nouvelles Lettres (18701923), Genève, Droz, 2001, p. 57.

${ }^{20}$ V. Pareto, La situation financière en Italie, «La Gazette de Lausanne », 5.12.1893, dans ibidem, Ecrits épars, Genève, Droz, 1974, p. 68. 
d'économie politique à l'Université de Lausanne, Pareto, lors de sa nomination, leur avait été présenté par le journal libéral ${ }^{21}$ comme une «acquisition brillante» de leur jeune université et un disciple de Walras. Il leur avait été signalé aussi comme «un libre-échangiste, espèce aujourd'hui rarissime ${ }^{22}$. Quelques jours après, un nouvel article ${ }^{23}$ ajoutait que Pareto était un «excellent mathématicien» et que, comme il possédait «à la fois le goût d'investigation de l'homme de science et l'aptitude à vulgariser qui fait le professeur», le Conseil d'Etat avait, somme toute, fait un bon choix.

Il y a deux manières de reconstituer la collaboration de Pareto à la Gazette. On peut suivre la chronologie de ses articles et, ainsi, approfondir la façon dont cette collaboration s'est déroulée dans les faits; cette méthode nous empêche toutefois de nous faire une idée suffisamment précise de la pensée que Pareto a offerte à ses lecteurs lausannois. Nous suivrons donc une seconde approche, en proposant une reconstruction rationnelle de ses articles, c'est-à-dire en donnant un ordre logique à leur contenu.

\section{a) Quelques jalons méthodologiques}

Notre reconstruction débute par les points de repère méthodologiques dont Pareto informe ses lecteurs. Pareto confirme que son approche est essentiellement celle adoptée par les sciences physiques et que John Stuart Mill a appelée «méthode déductive concrète»: il s'agit de déduire «de certains principes très généraux, tirés de l'expérience, ... des théories que l'on vérifie ensuite en examinant si elles expliquent bien tous les faits qui en dépendent ${ }^{24}$. Nous retrouvons donc l'expérience, conçue en tant qu'observation attentive et dépassionnée des faits, tant au début qu'à la fin des raisonnements scientifiques. Pareto invite les lecteurs de la Gazette à vérifier toujours, comme il le fait lui-même, si une théorie est confirmée par la pratique, en ouvrant deux «dossiers »: l'un pour les faits favorables à la théorie en question et l'autre pour les faits qui lui sont défavorables. Avant d'examiner «enfin, de quel côté penche la balance ${ }^{25}$.

De son choix méthodologique Pareto tire la conclusion qu'il ne faut pas prendre au sérieux les raisonnements a priori qui voudraient qu'une loi ait les effets souhaités par ses auteurs ${ }^{26}$ : au contraire, il faut toujours tenir compte du milieu où la loi est appliquée. Ainsi on ne sera pas surpris si, parfois, une loi a des conséquences opposées à celles visées (par exemple des mesures de persécution

${ }^{21}$ Gazette de Lausanne, «Université de Lausanne», 21.4.1893, p. 3.

${ }^{22}$ La Gazette de Lausanne du 2.11.1892, lors de sa campagne contre les députés protectionnistes français opposés à l'approbation du nouveau traité de commerce franco-suisse, avait déjà loué Pareto pour son article, Le imposte e la povera gente, «Il Secolo», 22-23.10.1892 (maintenant dans idem, Ecrits politiques. *Lo sviluppo del capitalismo (1872-1895), Genève, Droz, 1989), hostile au protectionnisme douanier et au militarisme.

${ }^{23}$ Gazette de Lausanne, «Université de Lausanne», 25.4.1893, p. 3.

${ }^{24}$ V. Pareto, «Discours de M. Vilfredo Pareto, professeur ordinaire d'économie politique, «Gazette de Lausanne», 23.10.1894, dans Idem, Marxisme et économie pure, op. cit., p. 117.

${ }^{25}$ V. Pareto, Des faits, «Gazette de Lausanne», 7.8.1897, dans idem, Ecrits épars, op. cit., pp. 127-131.

${ }^{26}$ V. Pareto, Matérialisme historique, «Gazette de Lausanne», 23.8.1902, idem, p. 155. 
qui profitent aux persécutés, comme cela s'est produit en Allemagne avec le parti du Centre catholique, pourtant menacé par Bismarck ${ }^{27}$ ) ou bien si la même loi génère, en des lieux différents, des effets opposés (l'impôt progressif en Prusse n'est pas dangereux pour la stabilité sociale parce qu'il est voté par ceux qui vont le payer; en France c'est le contraire qui est alors en train de se produire ${ }^{28}$ ). Pareto formule des critiques analogues à l'égard d'une évaluation des phénomènes économiques et sociaux qui se contenterait d'appliquer «quelque vague principe de morale», en renonçant à creuser leur complexité ${ }^{9}$.

En fait, et c'est là le point de départ de toute l'œuvre de Pareto, il suffit de réfléchir un peu pour se rendre compte de l' «étroite dépendance» existant entre tous les phénomènes sociaux. Ce n'est donc que pour faciliter leur étude que, au préalable, on examine ces phénomènes d'une manière isolée, mais en admettant toujours que, pour donner un avis sur ce qui arrive en pratique dans la société, il faut synthétiser ces études analytiques ${ }^{30}$.

\section{b) La définition et les limites de l'économie politique}

A partir de cette position méthodologique, Pareto peut alors annoncer à ses lecteurs qu'il y a deux types de théories économiques: celles qui s'accordent avec les faits, c'est-à-dire qui sont en mesure de reproduire ceux du passé et de prévoir ceux du futur, et celles qui ne correspondent pas aux faits et qui ne sont «d'accord qu'avec les sentiments humanitaires, socialistes et autres $»^{31}$.

L'économie politique qui est en accord avec les faits n'est que l'étude de tous les processus, connus par le biais de l'histoire et de la statistique, où les biens se transforment entre eux comme conséquence du «rapport de convenance entre une chose et un homme ${ }^{32}$ : cette étude n'aboutit qu'à classer ces approches et à essayer d'en tirer des lois générales ${ }^{33}$. Se trompent les gens qui pensent que les problèmes économiques sont fort simples et qui contestent que, pour leur étude, on fasse recours aux mathématiques: au contraire, ces problèmes sont très compliqués et, nonobstant l'aide apportée par les mathématiques, on a pu «à peine en effleurer l'étude ${ }^{34}$.

Dans le cadre de cette conception de l'économie politique, Walras, le prédécesseur de Pareto à la chaire d'économie politique de l'Université de Lausanne, a eu, notamment, le «principal (et) très grand mérite d'étudier, le premier ${ }^{35}$,

27 Ibidem.

${ }^{28}$ V. Pareto, L'impôt sur le revenu en France, «Gazette de Lausanne», 7.7.1903, dans idem, Lettres d'Italie, Genève, Droz, 1967, p. 145.

${ }^{29}$ V. Pareto, La spéculation, «Gazette de Lausanne», 11.11.1903, dans idem, Mythes et Idéologies, Genève, Droz, 1984, pp. 235-236.

${ }^{30}$ V. Pareto, Discours de M. Vilfredo Pareto, professeur ordinaire d'économie politique, art. cit., p. 119.

${ }^{31}$ V. Pareto, Crise future, «Gazette de Lausanne», 13.11.1907, dans idem, Lettres d'Italie, 1967, p. 162 .

${ }^{32}$ V. Pareto, Discours de M. Vilfredo Pareto, professeur ordinaire d'économie politique, art. cit., p. 117.

${ }^{33}$ Ibidem, p. 116.

${ }^{34}$ Ibidem.

${ }^{35}$ V. Pareto, L'œuvre scientifique de Léon Walras, Gazette de Lausanne, 8.1.1910, dans idem, Marxisme et économie pure, op. cit., p. 145. 
l'équilibre général, c'est-à-dire de mettre «en lumière le grand principe de la mutuelle dépendance des phénomènes économiques...»: pour atteindre ce but, il n'a pas pu se passer des mathématiques ${ }^{36}$.

Pareto ne perd pas l'occasion de rappeler à ses lecteurs que les lois de l'économie politique, en tant que science des moyennes, «comme beaucoup d'autres ${ }^{37}$, ne peuvent que reproduire la forme générale d'un phénomène et qu' «il serait ridicule de vouloir les étendre aux détails ${ }^{38}$. La théorie des crises économiques peut ainsi prévoir, par exemple, le début des périodes ascendante et descendante, mais pas la ruine de certaines entreprises durant la seconde et la prospérité d'autres entreprises durant la première ${ }^{39}$.

De surcroît, Pareto pense que l'économie politique ne doit pas empiéter sur les autres sciences sociales, telles que la morale et la religion, qui s'occupent des forces influençant les actions humaines. Ni, surtout, sur la science de l'utilité, qui vient d'être fondée par Gustave de Molinari et Herbert Spencer et qui étudie la propriété d'une chose «d'être, par son usage, favorable au progrès de l'humanité ${ }^{40}$.

Ceci dit, le cours d'économie politique que Pareto donne à Lausanne a pour but d'être utile à tous ses auditeurs, «quelque opinion qu'ils puissent avoir du reste sur les grands problèmes sociaux $»^{41}$.

\section{c) L'économie politique appliquée}

Pour Pareto l'économie politique théorique est le point de départ de l'étude appliquée à la réalité économique et, dûment développée en sociologie générale, de l'étude de la réalité sociale entière. La collaboration de Pareto avec la Gazette est parsemée d'exemples de l'application de l'économie politique à l'étude des faits économiques concrets. Cette intéressante série est ouverte par la courbe zurichoise de la répartition des revenus qui, basée sur les gros revenus, tombe très vite, contrairement à l'allure affichée par la courbe générale que Pareto vient de trouver pour ce phénomène ${ }^{42}$. L'article sur la fraude fiscale à Zurich, publié par la Gazette, explique bien cette énigme et suggère à Pareto l'idée que la fraude généralisée peut être présente dans tous les cas où on ne retrouve pas l'allure de la courbe générale susmentionnée ${ }^{43}$.

${ }^{36}$ Ibidem.

37 V. Pareto, Crise future, art. cit., p. 162.

38 V. Pareto, Les revenus des contribuables II, «Gazette de Lausanne», 21.12.1896, dans idem, Ecrits épars, op. cit., p. 127.

39 V. Pareto, Crise future, art. cit., p. 162.

${ }^{40}$ V. Pareto, Discours de M. Vilfredo Pareto, professeur ordinaire d'économie politique, art. cit., p. 117.

${ }^{41}$ Ibidem, p. 119.

${ }^{42}$ V. Pareto, Les revenus des contribuables I, «Gazette de Lausanne», 9 décembre 1896, dans idem, Ecrits épars, 1974, p. 125. Peu de jours après, suite d'une invitation de la Gazette à expliquer cette courbe sans avoir recours aux mathématiques, V. Pareto, «Les revenus des contribuables II», art. cit., pp. 126-127, présente sa découverte par le biais d'un graphique. La formule de la courbe est $\mathrm{N}=\mathrm{Ax}^{-\mathrm{a}}$ où $\mathrm{Y}$ est le nombre des contribuables ayant un revenu plus grand que $\mathrm{x}$; $\mathrm{A}$ et a sont des paramètres positifs. On en tire que $\mathrm{N}$ décroît d'une manière graduelle au fur en mesure que $\mathrm{x}$ croît.

${ }^{43}$ V. Pareto, Les revenus des contribuables I, art. cit., p. 125. 
Au début de $1899^{44}$, à l'occasion de la dévaluation du franc suisse face au franc français, Pareto rappelle à son public, comme l'une des théories économiques les «plus sûres », que cette situation est due à un faible taux d'intérêt qui implique un excès d'immobilisation de capitaux. Si on veut réévaluer le franc suisse, il faut donc procéder à une augmentation des taux d'intérêt suisses et, dans la foulée, accepter une réduction des immobilisations des capitaux. D'une façon analogue, la réévaluation, au printemps 1903, de la lire italienne vis-à-vis du franc français a eu lieu, selon Pareto, grâce à une administration publique plus économe qu'auparavant et à l'absence de mesures, de la part de la Banque d'Italie, contre les spéculateurs ${ }^{45}$. Mais, ajoute Pareto, ces faits ne suffisent pas à empêcher «messieurs les politiciens $»^{46} \mathrm{de}$ continuer, comme en France, à augmenter les dépenses électorales et les impôts, en ignorant que, de cette façon, ils font fuir les capitaux (la vraie «source de la production »): la baisse des titres de la dette publique française et la présence de plus en plus massive, auprès des banques étrangères, de titres déposés par les possédants français qui craignent d'être spoliés le démontrent ${ }^{47}$.

L'application la plus intéressante que Pareto offre à ses lecteurs concerne néanmoins les crises économiques, qu'il interprète comme de «simples mouvements rythmiques de l'agrégat social», à savoir comme une suite indéfinie de périodes «de dépression et d'activité ${ }^{48}$. Comme ces deux périodes «sont inséparables », il convient d'appliquer le nom de crise aux deux «moments », et non pas seulement à la phase descendante ${ }^{49}$. Quant à l'explication de la crise, elle ne peut se déduire de l'excès de consommation et de l'excès de production: même s'ils sont présents dans les périodes ascendante et descendante, ils «ne sont que des effets concomitants et des symptômes $»^{50}$. De la crise, selon Pareto, on ne peut donner qu'une description. Alors que l'on pensait autrefois que la prospérité et la dépression auraient duré «indéfiniment», on sait maintenant que ce n'est pas le cas. Les hommes d'affaires en ont tiré les conséquences et sont devenus un peu moins imprudents, ce qui a contribué à réduire l'intensité des crises ${ }^{51}$. En tout cas, la seule manière de les atténuer consiste dans la capacité «de ne pas se laisser griser [...] en des temps de prospérité, et de ne pas se laisser abattre [...] en des temps de dépression ${ }^{52}$. En outre, comme la Banque d'Angleterre a désormais l'habitude de le faire, il faudrait, pour rendre moins pénible la période descendante, hausser le taux d'escompte dès que ces signaux de crise que sont la dévaluation du change et les excès d'immobilisation font leur apparition ${ }^{53}$. p. 128

${ }^{44}$ V. Pareto, Le change, «Gazette de Lausanne», 7.1.1899, dans idem, Lettres d'Italie, 1967,

45 Ibidem.

46 Ibidem.

${ }^{47}$ Ibidem.

${ }^{48}$ V. Pareto, Le moment économique, «Gazette de Lausanne», 11.12.1905, dans idem, Lettres d'Italie, 1967, p. 152.

49 V. Pareto, La crise économique, «Gazette de Lausanne», 3.12.1906, ibidem, p. 154.

${ }^{50}$ V. Pareto, Crise financière ou économique, «Gazette de Lausanne», 20.9.1907, ibidem, p. 159.

${ }^{51}$ V. Pareto, Le moment économique, art. cit., p. 153.

52 Ibidem.

${ }^{53}$ V. Pareto, Le change, art. cit., p. 129. 
Chaque période de la crise est plus proche de sa fin «quand son intensité parâ̂tra plus grande ${ }^{54}$. La période ascendante ${ }^{55}$ fait augmenter la production et les prix de toutes les marchandises industrielles mais affecte aussi les autres phénomènes sociaux, par exemple en augmentant la disponibilité des contribuables à supporter des nouveaux impôts ${ }^{56}$. La période ascendante, remarque encore Pareto, «d'habitude» se termine «par un feu d'artifice d'émission de titres d'entreprises fantaisistes ${ }^{57}$, tandis que le début de la période descendante est annoncé par des signes comme la pénurie de charbon ${ }^{58}$.

Pareto offre ainsi à ses lecteurs des prévisions pour les crises de son époque. En décembre 1905, on est dans une période ascendante qui, selon Pareto, «aura probablement encore une certaine durée ${ }^{59}$. Une année après, Pareto peut montrer à ses lecteurs que sa prévision a été confirmée mais, tandis que l'on est au summum de la période ascendante, il peut désormais prédire qu'on n'est pas loin de sa fin et au début d'une nouvelle période descendante ${ }^{60}$. Et en été 1907, avec un brin d'orgueil ironique, Pareto rappelle à ses lecteurs que sa précédente prévision d'un début assez proche de la période descendante a été, elle aussi, confirmée par les faits. Pareto remarque aussi que les faits se sont chargés de démentir les deux critiques qui lui avaient été adressées. On lui avait en effet fait remarquer, d'une part, que le futur n'étant pas assimilable au passé, on n'aurait pas pu prévoir (comme prétendait le faire Pareto) les événements à partir de ce qui s'était déjà passé, et, d'autre part, que les trusts auraient été en mesure de désamorcer la crise $^{61}$. Or la période descendante a bel et bien commencé dans le pays même des trusts, c'est-à-dire les Etats-Unis ${ }^{62}$.

Dans ses articles consacrés à la crise économique, Pareto remarque aussi l'existence d'une tendance continue au renchérissement dont la cause générale réside dans une augmentation de la population moins rapide que celle de la richesse. $\mathrm{Ce}$ qui a comme conséquence de dévaloriser l'argent vis-à-vis du travail ${ }^{63}$. Parmi les causes secondaires du renchérissement, on trouve «les lois dites de protection sociale, les grèves très fréquentes, un changement dans les habitudes des populations, qui veulent travailler de moins en moins et gagner de plus en plus » ${ }^{64}$. Ces derniers changements favorisent de plus la hausse des taux d'intérêt, autant qu'ils diminuent la production des capitaux tout en en stimulant, en même temps, la demande, afin de remplacer une main d'œuvre désormais plus chère ${ }^{65}$. Dans ce

${ }^{54}$ V. Pareto, Le moment économique, art. cit., p. 153.

55 Ibidem, p. 152.

56 Ibidem, p. 153.

57 V. Pareto, Période descendante, «Gazette de Lausanne», 5.6.1907, dans idem, Ecrits épars, op. cit., p. 175.

${ }^{58}$ V. Pareto, Crise financière ou économique, art. cit., p. 159.

59 V. Pareto, Le moment économique, art. cit., p. 152.

${ }^{60} \mathrm{~V}$. Pareto, La crise économique, art. cit., p. 154.

${ }^{61}$ V. Pareto, Période descendante, art. cit., p. 174.

${ }^{62}$ Ibidem, p. 174.

${ }^{63}$ V. Pareto, La crise économique, art. cit., p. 154.

${ }^{64}$ V. Pareto, Crise financière ou économique, art. cit., p. 161.

${ }^{65}$ Ibidem, p. 161. 
cadre général, les crises impriment au mouvement de renchérissement «une marche ondulée », en l'accélérant pendant la période ascendante et en le ralentissant pendant la période descendante ${ }^{66}$.

D'une manière un peu paradoxale enfin, Pareto, en automne 1903, remarque que, si les spéculateurs n'ont pas une bonne renommée, le gouvernement allemand, qui venait de durcir les règlements boursiers, démontre que si l'on élimine la spéculation, on élimine aussi le progrès économique ${ }^{67}$. En fait, chaque individu, selon Pareto, fait une utilisation permanente d'une partie seulement de son épargne et entend conserver le reste «en vue de besoins prévus ou imprévus ${ }^{68}$. L'organisation financière moderne a permis de mobiliser l'épargne et a encouragé son utilisation complète, ce qui facilite la production ${ }^{69}$. La spéculation est donc bien l'un des rouages les plus utiles de cette organisation et permet à ceux qui ont placé leur épargne en titres de la récupérer à chaque moment «sans trop y perdre, au moins en des temps ordinaires ${ }^{70}$. Et quand bien même les spéculateurs ne sont pas des philanthropes... Il y en a aussi de malhonnêtes qui, comme partout dans la société, provoquent parfois des ruines, «comme les chemins de fer qui tuent des voyageurs $»^{71}$.

\section{d) Les définitions économiques du libéralisme et du socialisme}

L'économie politique permet également à Pareto d'aborder le grand débat de politique économique de son époque, qui oppose le libéralisme au socialisme. La liberté économique demande qu'on laisse chacun faire ce qu'il veut de son épargne et de son travail avec la certitude, soutenue par la connaissance historique et par l'observation attentive des faits courants, que si l'on respecte le droit de propriété, on assure le maximum de bien-être à la sociétét ${ }^{72}$. Cette définition a été exprimée sous forme de théorème par Walras quand celui-ci a démontré que ce sont bien les prix fixés par la libre concurrence qui assurent le maximum d'utilité pour la société ${ }^{73}$. Mais Walras est lui-même critiqué par Pareto quand il entend, avec une «généreuse impatience», appliquer sa théorie économique à la solution de problèmes pratiques. Selon Pareto, cette approche demande encore un travail théorique considérable ${ }^{74}$.

Pareto observe, en passant, que les soucis des conseils d'administration de certaines entreprises nationalisées, lorsqu'il s'agit d'éviter la disparition de leurs capitaux, démontrent bien que la conservation du capital «n'est pas une sinécure» et que, de ce fait, le capitaliste «n'est pas tout à fait un parasite ${ }^{75}$. Enfin, contrai-

66 V. Pareto, La crise économique, art. cit., p. 155.

${ }^{67}$ V. Pareto, La spéculation, art. cit., p. 234.

${ }^{68}$ Ibidem.

${ }^{69}$ Ibidem, p. 235.

${ }^{70}$ Ibidem.

71 Ibidem.

${ }^{72}$ V. Pareto, Mieux vaut un franc ennemi, «Gazette de Lausanne», 3.12.1895, dans idem, Inédits et Addenda, Genève, Droz, 2005, p. 188.

${ }^{73}$ V. Pareto, Discours de M. Vilfredo Pareto, professeur ordinaire d'économie politique, art. cit., p. 116.

${ }^{74}$ V. Pareto, L'œuvre scientifique de Léon Walras, art. cit., p. 145.

75 V. Pareto, La mine aux mineurs, «Gazette de Lausanne», 4.2.1901, dans idem, Mythes et idéologies, op. cit., p. 185. 
rement à ce dont on les accuse, les économistes libéraux préfèrent les mesures qui moralisent le peuple plutôt que celles qui l'enrichissent, de la même manière qu'ils estiment que la moralité amène la richesse, tandis que l'immoralité alimente la pauvreté ${ }^{76}$.

Le socialisme, au contraire, se prononce pour le changement de la «répartition naturelle de la richesse » par le biais de la loi. Le socialisme pratiqué par les bourgeois et le socialisme pratiqué par les prolétaires ne diffèrent en réalité que par les bénéficiaires et les victimes de cette nouvelle répartition, c'est-à-dire les pauvres dans le cas du socialisme bourgeois et les riches dans le cas du socialisme populaire ${ }^{77}$. En définitive, toutes les sortes du socialisme provoquent la «ruine de la société ${ }^{78}$. Et il va sans dire que, s'ils veulent être cohérents avec leur idéaux, les libéraux ne doivent pas se manifester pour quelque forme de socialisme que ce soit... ${ }^{79}$

\section{- Le socialisme mis en pratiqué par la bourgeoise italienne par le biais de l'Etat}

Pareto examine, pour ses lecteurs lausannois, les expériences les plus importantes et les plus dangereuses du socialisme mis en pratique, selon lui, par la bourgeoisie italienne et française. Le premier article de Pareto dans la Gazette concerne la faillite de la deuxième banque italienne de l'époque, le Credito Mobiliare de Florence. Pareto ${ }^{80}$ y souligne que les nombreuses banques et sociétés de commerce italiennes qui sont en faillite auraient pu prospérer si elles s'étaient contentées de faire leur métier. Mais toutes ont voulu travailler avec les politiciens en espérant participer à l'exploitation des contribuables pratiquée à partir de l'année 1876, lorsque la gauche historique est arrivée au pouvoir. Or les espoirs des banquiers et des hommes d'affaires italiens étaient vains: les contribuables sont sortis épuisés et ni les politiciens, «ni leurs complices», ne peuvent plus en profiter. Il s'ensuit que l'argent versé par les banquiers pour bénéficier des faveurs des politiciens «est presque entièrement perdu»: la faillite est inévitable.

La crise bancaire italienne a fini par frapper le système de l'émission de monnaie et Pareto commente ${ }^{81}$ pour ses lecteurs le verdict auquel vient d'aboutir le procès à Bernardo Tanlongo, patron de la Banca Romana. Selon Pareto, l'absolution octroyée est semblable à celle dont ont bénéficié, à la même époque, les accusés du célèbre scandale français du Panama: les jurés ont estimé injustes de punir seulement les complices alors que les plus «grands coupables », eux, ne sont pas inquiétés.

Ce scandale ne termine toutefois pas une crise, selon Pareto, désormais inhérente au système. La mauvaise administration du Banco di Napoli, l'une des trois banques d'émission restantes après la création de la Banque d'Italie (en 1893,

${ }^{76}$ V. Pareto, Mieux vaut un franc ennemi, art. cit., p. 189.

77 Ibidem, p. 188.

78 Ibidem.

79 V. Pareto, La situation en Italie, «La Gazette de Lausanne», 28.5.1898, dans idem, Lettres d'Italie, op. cit., pp. 121-124, p. 121.

80 V. Pareto, La situation financière en Italie, art. cit., p. 68.

${ }^{81}$ V. Pareto, Le verdict Tanlongo, «Gazette de Lausanne», 2.8.1894, dans idem, Inédits et Addenda, op. cit., pp. 130-132. 
après le scandale de la Banca Romana), se poursuit selon l'habitude, mis à part quelques changements de forme: le gouvernement italien, en fait, impose au Banco d'escompter des traites fort douteuses ou lui arrache une bonne partie de l'or qui était dans ses caisses en le remplaçant par «certains morceaux de papier ${ }^{82}$. En somme, le Banco sert à financier les hommes politiques et, comme «les mêmes causes manquent rarement de produire les mêmes effets », Pareto prévoit que la mauvaise administration du Banco aboutira à une faillite, comme cela a été le cas pour la Banca Romana ${ }^{83}$.

Pareto ne se lasse pas d'observer le phénomène bancaire italien et, quelques années plus tard, il peut annoncer que, de deux dossiers pour et contre la théorie «des bienfaits de l'intervention de l'Etat dans l'administration des banques», celui des faits favorables continue à rester vide tandis que celui des faits défavorables déborde. L'énième cas répertorié concerne un scandale bancaire qui a eu lieu à Côme et dont les actionnaires de la Banque d'Italie ont été victimes, essentiellement parce que le directeur général de celle-ci avait été nommé non par eux, mais par le gouvernement ${ }^{84}$.

Au début de $1894^{85}$, Pareto renseigne ses lecteurs sur les impôts italiens auxquels il attribue la difficile situation des contribuables de ce pays. Celle-ci a débouché non seulement sur une crise des recettes fiscales de l'Etat mais aussi, et surtout, sur de sanglantes révoltes populaires, notamment en Sicile. Cette situation est due à la «mégalomanie d'un petit nombre d'individus... [et aux] malversations et [aux] vols de politiciens sans vergogne». L'excès d'étatisme est également la cause des émeutes milanaises du mai $1898^{86}$. Il va sans dire que la seule solution possible aux maux de l'Italie est de revenir à la liberté économique introduite par Cavour et de réduire les dépenses publiques, pour réduire ensuite les impôts ${ }^{87}$. Le gouvernement propose au contraire une mesure traditionnellement prônée par les mêmes socialistes qu'il met en prison: il s'agit de travaux publics, qui vont augmenter les dépenses publiques, donc les impôts et/ou la dette publique ${ }^{88}$.

Au début de 1899, Pareto évoque le décret royal italien qui vient de punir, avec amende et prison et sans rien leur garantir en retour, les travailleurs des chemins de fer, des postes, des télégraphes et de l'éclairage qui osent de se concerter pour se mettre en grève ${ }^{89}$. Selon Pareto, ce serait le retour de la servitude si, par ce biais, les patrons, d'une manière parfaitement légale, pouvaient décider de réduire les salaires de leurs employés tandis que ces derniers n'auraient plus le droit de refuser de

${ }^{82}$ V. Pareto, Une banque malheureuse, «Gazette de Lausanne», 13.2.1895, dans idem, Lettres d'Italie, op. cit., pp. 115-117.

${ }^{83}$ Le Banco réussira à survivre à la crise mentionnée.

${ }^{84}$ V. Pareto, Des faits, art. cit., p. 130.

${ }^{85}$ V. Pareto, Les impôts en Italie, «Gazette de Lausanne», 8.1.1894, dans idem, Inédits et Addenda, op. cit., p. 126.

${ }^{86}$ V. Pareto, La situation en Italie, art. cit., p. 121.

${ }^{87}$ Ibidem, p. 124.

88 Ibidem.

${ }^{89}$ V. Pareto, Le socialisme d'Etat en Italie, «Gazette de Lausanne», 29.1.1899, dans idem, Ecrits épars, op. cit., p. 147. 
travailler pour ces nouveaux salaires ${ }^{90}$. A ce propos, Pareto partage l'idée de l'historien économique anglais libéral Thorold Rogers et selon laquelle il faut permettre à tout le monde, capitalistes et ouvriers compris, de s'associer pour mieux défendre ses intérêts ${ }^{91}$, comme cela s'est déjà produit, sans conséquences catastrophiques, en Angleterre $^{92}$. Sauf à demander aux travailleurs des services publics, quand ils font usage de leur droit de grève, de donner leur congé par avance...

Etant donné qu'interdire la grève à certaines catégories de travailleurs seulement n'a aucun sens, peut-être que ce droit devra-t-il être enlevé à tous mais, «comme il serait monstrueux de laisser ainsi les ouvriers à la merci de leurs employeurs », il faudra au moins fixer, par la loi, le salaire et la durée du travail: on aura ainsi «une organisation complète du socialisme d'état ${ }^{93}$.

\section{- Le radico-socialisme en France}

Pour ce qui concerne la France de la fin du siècle, Pareto est de l'avis que les amis bourgeois des socialistes, tels Léon Bourgeois et Pierre Waldeck-Rousseau, sont «plus malfaisants que les socialistes eux-mêmes» puisqu'ils sont responsables, et de l'augmentation continue des dépenses publiques (et donc des impôts), et de l'affaiblissement de la résistance anti-socialiste, notamment dans la magistrature et l'armée ${ }^{94}$. Et comme La Fayette au temps de la Révolution, Waldeck-Rousseau se trompe quand il pense pouvoir contrôler ses alliés socialistes, par exemple en les associant à la lutte contre un prétendu «péril clérical»: le lion socialiste finira par dévorer le radical qui pensait l'avoir dompté... ${ }^{95}$

Pareto estime donc que les socialistes français, capables d'entraîner même ceux qu'ils veulent dérober, ont toutes les chances de leur côté. Le seul doute concerne en fait l'usage qu'ils feront de leur victoire ${ }^{96}$. Pareto, à propos du cas français, précise aussi qu'il ne faut pas confondre le socialisme humanitaire avec le marxisme, qui est une doctrine scientifique, bien qu' «au moins en partie» erronée ${ }^{97}$ : on ne peut donc admettre que le marxisme a été bridé seulement par la présence de quelques humanitaires au gouvernement ${ }^{98}$. Et surtout, on ne peut penser que les masses populaires continueront à se laisser tromper: elles exigeront un jour ou l'autre que les promesses soient remplies et, alors, comme les Montagnards ont succédé aux Girondins, après «les humanitaires» viendront «les collectivistes pratiques $\gg{ }^{99}$.

\footnotetext{
${ }^{90}$ Ibidem, pp. 147-148.

${ }^{91}$ Ibidem, p. 148.

92 Ibidem.

${ }^{93}$ Ibidem

94 V. Pareto, Une expérience sociale, «Gazette de Lausanne», 19.11.1900, dans idem, Mythes et idéologies, op. cit., p. 168.

${ }^{95}$ Ibidem, p. 169.

${ }^{96}$ Ibidem, p. 170.

97 V. Pareto, Deux socialismes, « Gazette de Lausanne», 27.1.1903, ibidem, p. 215.

98 Ibidem.

99 Ibidem.
} 


\section{e) Le déclin du libéralisme}

C'est en Suisse en général, et dans Pays de Vaud en particulier, que Pareto, dès la moitié des années 1890, pense apercevoir les derniers remparts du libéralisme. Car l'histoire est là pour nous démontrer que l'ordre est «le premier de tous les besoins des sociétés humaines ». Pareto en tire la conclusion que si la démocratie n'est pas en mesure de joindre la liberté à l'ordre, elle cédera sa place au despotisme: mais la démocratie suisse lui semble à l'abri de ce danger ${ }^{100}$.

Pareto remarque aussi que la plus grande erreur de la politique pratique est celle de croire à l'existence de lois qui puissent «guérir toutes sortes de maux », en ignorant, notamment, «les qualités morales et intellectuelles du peuple» auquel les lois sont appliquées. A titre d'exemple, Pareto remarque qu'en Italie et dans le canton de Vaud, il y a plus ou moins les mêmes lois qui imposent l'instruction obligatoire et garantissent la propriété immobilière: cependant, ces lois sont respectées d'une manière scrupuleuse dans le Pays de Vaud et violées d'une manière flagrante en Sicile $\mathrm{e}^{101}$.

Pareto relève un dernier succès libéral en novembre 1902 lorsque, dans l'Europe protectionniste, les agriculteurs danois, non seulement ne demandent pas de protection douanière, mais la refusent ${ }^{102}$ en gardant à l'esprit que si l'on restreint les importations, on restreint aussi les exportations ${ }^{103}$.

Le déclin irréversible du libéralisme, déjà anticipé par l'attitude de la bourgeoise radicale française, est confirmé de la manière la plus claire en 1903, à l'occasion du fort probable abandon du libre-échange par l'Angleterre, le pays qui avait inventé et pratiqué cette politique. En général, le libéralisme " est une doctrine optimiste car elle suppose que les hommes pourront renoncer à se spolier les uns les autres », mais les faits indiquent bien que les conditions de sa réalisation ne sont pas encore remplies ${ }^{104}$. Bien que le changement survenu en Angleterre puisse paraître étonnant, il n'est, selon Pareto, que la conséquence d'une liberté des échanges qui ne peut survivre quand toutes les autres libertés n'existent plus ${ }^{105}$. En fait, si les libéraux anglais avaient défendu le libre-échange, ils auraient été en contradiction avec tous leurs agissements récents qui ont abouti, depuis une douzaine d'années, «à restreindre les libertés » anglaises, en favorisant toutes sortes des régulations étatiques, au nom de l'hygiène et de la tempérance, les syndicats ouvriers, qui ont provoqué des renchérissements bien plus considérables que les tarifs douaniers, ou encore le socialisme municipal ${ }^{106}$.

Pareto ajoute enfin que Joseph Chamberlain, le leader du fair trade, s'est montré fort habile en liant le renoncement au libre-échange à l'établissement des

${ }^{100}$ V. Pareto, Discours de M. Vilfredo Pareto, professeur ordinaire d'économie politique, art. cit., p. 115 .

${ }^{101}$ Ibidem

${ }^{102}$ V. Pareto, Un Pays exceptionnel, «Gazette de Lausanne», 26.11.1902, dans idem, Lettres d'Italie, op. cit., p. 138.

${ }^{103}$ Ibidem, p. 139.

104 V. Pareto, L'éclipse de la liberté, «Gazette de Lausanne», 8.6.1903, dans idem, Mythes et idéologies, op. cit., p. 224.

105 Ibidem.

${ }^{106}$ Ibidem, p. 225. 
pensions pour les vieillards: en leur rendant une petite partie de ce que leur prendront l'Etat et les producteurs dûment protégés, il va ainsi obtenir l'appui des gens qui payeront les frais de la protection ${ }^{107}$. Inutile de dire que, si ces derniers n'avaient pas été ponctionnés par les protections douanières, et s'ils n'avaient été persuadés du bien-fondé de la tutelle instaurée par l'Etat à leur égard ${ }^{108}$, ils auraient pu pourvoir eux-mêmes à leurs pensions !

\section{f) Vers l'instauration du socialisme populaire pratique, c'est-à-dire vers des nouvelles élites?}

Ainsi convaincu que le libéralisme n'a plus son mot à dire, Pareto continue à observer le phénomène socialiste avec la sérénité que lui procure l'abandon définitif de son militantisme libéral, au profit de la science sociale à l'essor de laquelle il compte donner de nouvelles contributions originales.

Les essais de socialisme populaire pratique, que Pareto note, pourraient aboutir tout simplement à la création de nouveaux privilégiés même si, cette fois, ils proviennent de la classe ouvrière. Pareto analyse en réalité les expériences socialisantes de la mine française de Monthieux qui, acquise et gérée à partir de 1891 par les mineurs eux-mêmes, avait échappé à la faillite en 1895 déjà ${ }^{109}$. La moitié des mineurs actionnaires avaient donné leur démission et cédé leurs actions: Pareto souligne ainsi ce nouveau cas de sélection entre des gens qui, en étant tous propriétaires, préfèrent, les uns, être à nouveau embauchés avec un salaire régulier et, les autres, sacrifier «le présent en vue de l'avenir; ce sont des spéculateurs ${ }^{110}$. A Monthieux, ces ouvriers spéculateurs embauchent eux aussi des salariés, mais avec le titre d' «auxiliaires ». Et Pareto d'ironiser sur ce changement de nom qui fut le seul résultat de « la grande transformation collectiviste [...] on aurait bien mauvaise grâce à s'y refuser $»^{111}$.

En tout cas, la mine, gérée par les ouvriers spéculateurs selon le système capitaliste, c'est-à-dire sans l'indiscipline de ses premières périodes, prospère ${ }^{12}$. Pareto en tire l'enseignement que «c'est aussi juste qu'utile» que la production ne soit dirigée que par ceux qui pratiquent «le travail, l'épargne, le sacrifice de soi [...] quel que soit leur point de départ»: en fait, la société se ruinerait si elle ne pouvait pas compter sur une élite pareille ${ }^{113}$.

En général ${ }^{114}$ «la formation de nouvelles classes privilégiées », qui, au début du siècle, sont représentées par certaines catégories d'ouvriers (par exemple, les lamineurs, les mineurs, les employés de chemin de fer ou de tramway), a toujours été observée. A la suite de situations particulièrement favorables, celles-ci ont

\footnotetext{
${ }^{107}$ Ibidem.

108 Ibidem.

${ }^{109}$ V. Pareto, La mine aux mineurs, art. cit., p. 184.

${ }^{110}$ Ibidem, p. 185.

111 Ibidem.

${ }^{112}$ Ibidem, p. 186.

${ }^{113}$ Ibidem, p. 187.

${ }^{114}$ V. Pareto, Nouvelles castes sociales, «Gazette de Lausanne», 25 9.1902, dans idem, Mythes et idéologies, op. cit., p. 204.
} 
réussi à bénéficier de très bons salaires. Enviées par les petits bourgeois et les paysans, elles sont défendues par des syndicats qui sont parvenus à monopoliser le marché du travail en limitant son accès à leurs membres et en obligeant les patrons à n'embaucher que ces derniers ${ }^{115}$. Ces actions syndicales sont favorisées, pour des raisons électorales, par les gouvernements qui, ainsi, ont vis-à-vis des ouvriers syndiqués la même relation qui, autrefois, poussait les gouvernements à protéger la noblesse ${ }^{116}$. Selon Pareto, ces nouveaux privilèges seront brisés par une révolution pacifique ou violente et, immédiatement après, de nouveaux s'en créeront: dans ce cas de figure, pour Pareto, il faudra toutefois une longue période avant que ces privilèges ouvriers ne puissent être anéantis ${ }^{117}$. Le grand économiste relève cependant qu'en France, en revanche, les socialistes ne doutent pas que leur révolution sera violente et qu'une fois achevée, ils pourront se passer «des parasites de la société bourgeoise » qui prétendent les représenter au gouvernement ${ }^{118}$.

\section{g) Les entraves au succès du socialisme populaire: l'insupportable inefficacité de son organisation économique, ses clivages idéologiques et le possible début de la réaction bourgeoise}

Le succès du socialisme populaire, en tout cas, n'est pas garanti. Pareto passe en revue toute une série d'entraves que ce mouvement rencontre. Il y a avant tout des difficultés de type économique. Pareto observe que, dans la deuxième moitié du XIX ${ }^{\mathrm{e}}$ siècle, les dépenses publiques ont beaucoup augmenté mais moins que la production, avec la conséquence que le bien-être matériel des gens a crû d'une manière remarquable ${ }^{119}$. Mais, comme on ne prévoit pas de nouveaux progrès technologiques aussi importants que ceux qui ont eu lieu dans les chemins de fer ou dans la grande industrie, l'augmentation des dépenses publiques (qui, pour Pareto, ne sont que du gaspillage à finalité électorale) va dépasser celle de la richesse, avec une diminution du bien-être matériel à la clé. Cette évolution pourrait donner lieu à une crise politique susceptible de changer l'actuel régime politique européen de plus en plus influencé par le socialisme populaire ${ }^{120}$.

Dans le même sens, après avoir commenté d'une manière sarcastique l'allure catastrophique des cotations de certains trusts américains à la bourse de New York, Pareto remarque que les trusts jouent le même rôle économique que les syndicats ouvriers. Il en déduit que c'est seulement si «on est aveuglé par la passion, ou l'intérêt » que l'on peut, d'un côté, juger d'une manière favorable les résultats obtenus par les syndicats et, de l'autre, condamner ceux des trusts ${ }^{121}$. En

${ }^{115}$ Ibidem, p. 205.

${ }^{116}$ Ibidem.

${ }^{117}$ Ibidem, p. 206.

${ }^{118}$ V. Pareto, Opinions provinciales, «Gazette de Lausanne», 28.10.1902, dans idem, Ecrits épars, op. cit., p. 159.

${ }^{119}$ V. Pareto, L'impôt sur le revenu en France, art. cit., p. 146.

${ }^{120}$ Ibidem, p. 146.

${ }^{121}$ V. Pareto, Trusts et Syndicats, «Gazette de Lausanne», 29.8.1903, dans idem, Mythes et Idéologies, op. cit., p. 233. 
fait, ces deux organisations n'ont tous deux obtenu que des résultats négatifs. Les syndicats australiens le montrent: en protégeant le travail national, ils ont provoqué la multiplication des faillites, une baisse de la production de moutons et une baisse de la production d'or dans les mines, pourtant seule en mesure de payer les salaires «fantastiques» imposés par la loi et les syndicats ${ }^{122}$. De cette façon, les capitaux anglais qui financent l'économie australienne sont de moins en moins rémunérés et les perspectives sombres que propose l'Australie n'arrangent rien: voilà une démonstration supplémentaire de la capacité des socialistes «à dévorer le capital», sans «le reconstituer $»$ ensuite ${ }^{123}$.

Mais le fait que le socialisme populaire rime avec une drastique réduction de l'efficacité du système économique, et donc du bien-être de la société, n'est pas seulement démontré par les excès des dépenses publiques et par les excès du pouvoir syndical. En fait, il est dû surtout à l'expérience du socialisme d'Etat en vigueur en Italie, un socialisme soutenu a contrario par la loi, «expression abrégée d'un nombre immense de faits», selon laquelle l'homme est poussé à l'action par des mobiles «automatiques, internes», et non pour des motifs «coercitifs, externes». Autrement dit, que «l'intérêt privé est le plus puissant ressort qui puisse être mis en œuvre pour pousser l'homme au travail $\gg{ }^{124}$. Ainsi le socialisme populaire devra probablement confier «toute la production» à un sujet aussi inefficace que l'Etat et il ne fait aucun doute que le peuple concerné «devra jeûner au moins six jours la semaine ${ }^{125}$.

En France, Jaurès, Millerand et leurs amis n'ignorent bien sûr pas que, pour satisfaire les appétits qu'ils ont suscités, il ne suffit pas d'octroyer des monopoles à l'Etat ${ }^{126}$. Ces socialistes bourgeois finiraient par être accusés, par leur propre parti, de beaucoup promettre et ne rien tenir ${ }^{127}$, d'autant qu'ils semblent craindre eux-mêmes «l'avènement de la société collectiviste»: «A peine ils se rapprochent du pouvoir [...] les voit-on s'occuper de tout autre chose que de réaliser leurs théories », conclut Pareto... ${ }^{128}$ C'est pourquoi Pareto prévoit, en été 1906, que le socialisme sera supplanté par le syndicalisme révolutionnaire ${ }^{129}$. Il ne faut pas confondre ce syndicalisme avec l'anarchie, nonobstant qu'ils prêchent, tous les deux, la violence: seul le premier, collectiviste au contraire de l'autre, «peut arriver à organiser une société ${ }^{130}$.

Théoriquement, le syndicalisme souhaite une reprise de l'idée de Marx en faveur d'une lutte des classes «implacable, féroce, sauvage», avec pour conséquence non pas une évolution, mais une révolution de la société ${ }^{131}$. «Au point de

\footnotetext{
${ }^{122}$ Ibidem.

123 Ibidem.

${ }^{124}$ V. Pareto, Des faits, art. cit., p. 129.

${ }^{125}$ Ibidem, p. 131.

${ }^{126}$ V. Pareto, Socialisme scolaire, «Gazette de Lausanne», 28.2.1903, dans idem, Mythes et Idéologies, op. cit., p. 219.

${ }^{127}$ V. Pareto, Socialisme bourgeois, «Gazette de Lausanne», 26.1.1904, ibidem, p. 246.

128 Ibidem.

${ }^{129}$ V. Pareto, Le syndicalisme, «Gazette de Lausanne», 7.9.1906, dans idem, Mythes et Idéologies,

${ }^{130}$ Ibidem.

${ }^{131}$ Ibidem.
} op. cit., p. 266. 
vue pratique, le syndicalisme représente les appétits qu'a excités, sans pouvoir les satisfaire, le socialisme humanitaire et décadent », chevauché à gauche, en France, en Allemagne ou en Italie, par ce nouveau parti qu'est la social-démocratie ${ }^{132}$.

Selon Pareto, les articles sur la violence que Georges Sorel vient de publier dans le Mouvement socialiste décrivent bien la nature du syndicalisme révolutionnaire $^{133}$. Selon Sorel, les violences du prolétariat ne sont que des actes de guerre et le syndicalisme veut, comme Marx, que la révolution sociale n'aboutisse pas «à remplacer une minorité gouvernante par une autre minorité». Mais, pour Pareto, ce dernier souhait n'est qu'un désir ${ }^{134}$. Un autre auteur syndicaliste, Hubert Lagardelle, montre bien que les syndicalistes veulent en réalité détruire l'Etat en le désorganisant, au lieu de le conquérir comme le font les socialistes parlementaires ${ }^{135}$. Pareto, comme «toute personne qui étudie froidement les faits, sans parti pris et sans passions », relève que la désorganisation prônée par les syndicalistes est en route et que «l'avenir... sera certainement tout autre que celui que désirent et rêvent les pacifiques humanitaires ${ }^{136}$.

Face à ce front socialiste conquérant, mais de moins en moins uni, la bourgeoise, sauf en France, donne, de temps en temps, des signes de ressaisissement. Le nouveau tarif allemand, adopté début 1903, doit être considéré non seulement du point de vue économique, mais du point de vue social, vu qu'il est fondamental pour l'Etat de pouvoir compter sur des classes agricoles prospères: n'est-ce pas ces dernières qui génèrent les meilleurs citoyens et les meilleurs soldats ${ }^{137}$ ? Ainsi, selon Pareto, les pertes causées en Allemagne par la protection douanière sont largement compensées par les avantages que cette dernière procure aux agriculteurs $^{138}$. En revanche, les socialistes allemands, responsables du renchérissement par le biais de leurs syndicats, sont favorables au libre-échange seulement parce qu'ils savent que l'aggravation de la condition des agriculteurs pourra les aider à briser la seule vraie résistance qui s'oppose à leur action ${ }^{139}$. Si le gouvernement allemand réussit à contrecarrer la désagrégation suscitée par les socialistes, il s'appropriera «l'hégémonie de l'Europe (et) comme le monde a toujours appartenu aux forts, il leur appartiendra longtemps encore ${ }^{140}$.

En janvier 1904, Pareto note aussi un double succès patronal, en Saxe, où les patrons se sont solidarisés et le gouvernement a étouffé les violences provoquées par les grévistes ${ }^{141}$. Et de prévoir que les patrons vont s'unir de plus en plus entre eux mais qu'ils continueront aussi «à fronder le gouvernement», en favorisant, ainsi, les syndicats ouvriers ${ }^{142}$.

\footnotetext{
${ }^{132}$ Ibidem.

${ }^{133}$ Ibidem.

${ }^{134}$ Ibidem.

135 Ibidem.

${ }^{136}$ Ibidem, p. 268.

${ }^{137}$ V. Pareto, Le tarif douanier allemand, «Gazette de Lausanne», 24.12.1902, dans idem, Lettres d'Italie, op. cit., p. 141.

${ }^{138}$ Ibidem.

${ }^{139}$ Ibidem, p. 142.

${ }^{140}$ Ibidem.

${ }^{141}$ V. Pareto, Socialisme bourgeois, art. cit., p. 246.

${ }^{142}$ Ibidem.
} 
Pareto repère aussi les signes d'une certaine réaction bourgeoise de type intellectuel. Il remarque ainsi qu'à Florence vient d'être fondée une revue, Il Regno, qui a des buts explicitement antihumanitaires: Pareto estime «que la vérité se trouve en un juste milieu» mais si on ne vise, comme c'est le cas de cette revue, que des buts pratiques, alors «aux doctrines exagérées en un sens (les humanitaires), il est souvent indispensable d'opposer des doctrines exagérées en un autre ${ }^{143}$. Ne voulant pas «scandaliser» les lecteurs de la Gazette, Pareto se borne à mentionner que la revue florentine ne croit pas à «la paix par le droit», qu'elle ne sympathise pas avec les faibles mais avec les «vainqueurs de la lutte pour la vie», qu'elle souhaite une bourgeoisie capable de rendre les coups à ses adversaires et, enfin, qu'elle sait «aussi prendre l'offensive ${ }^{144}$.

\section{CONCLUSION}

La recherche, que nous venons de présenter, semble confirmer que la collaboration avec la Gazette permet à Pareto d'exposer au grand public lausannois les idées qu'il mûrit dans son activité universitaire, dans la capitale vaudoise. Après avoir dévoilé, dès le début, ses prémisses méthodologiques, Pareto porte son attention sur la phénoménologie sociale qu'il observe à travers l'économie politique renouvelée qu'il enseigne, et notamment à travers le théorème de l'optimalité économique de la liberté. Il offre à ses lecteurs des articles très clairs sur la nature et la prévisibilité des crises économiques.

Mais c'est grâce à l'analyse amère, également proposée dans la Gazette, de l'éclipse du libéralisme que Pareto se persuade que le schéma rationnel de l'économie politique n'explique qu'une petite partie des actions humaines. En fait, «ce ne sont pas des raisonnements qui poussent les hommes à l'action, ce sont les intérêts, les sentiments, les passions, pourvu, bien entendu, que ces hommes aient la force d'agir ${ }^{145}$ : ce sera le début de la sociologie de Pareto.

Dans les colonnes de la Gazette, Pareto consigne ses réflexions sur les côtés économiques décevants de l'expérience socialiste que l'humanité, ignorante de ce que suggère la raison, veut, coûte ce que coûte, réaliser. Mais peut-être le socialisme se soldera-t-il aussi, non par une catastrophe économique, mais tout simplement par l'essor, dans le respect de la propriété privée, de nouvelles élites en provenance du prolétariat: et comme le progrès social ne dépend que des nouvelles élites, cette possible évolution socialiste se révélera moins inquiétante !

\footnotetext{
${ }^{143}$ Ibidem.

${ }^{144}$ Ibidem.

${ }^{145}$ V. Pareto, Une expérience sociale, art. cit., p. 170.
} 